\title{
Review of Fournier's Gangrene
}

\author{
${ }^{1}$ Tabowei B I, MBBS, FMCS. ${ }^{2}$ Kombo BB, MBBS, FRCS. \\ ${ }^{1 .}$ Niger Delta University Teaching Hospital, Okolobiri,Bayelsa ,Nigeria. \\ ${ }^{2}$ University Of PortHarcourt Teaching Hospital, Port Harcourt,Rivers state, Nigeria.
}

\begin{abstract}
This paper reviews 15 cases of Fournier's gangrene (FG) seen in our facility (NDUTH) over a period of 5 years .2002-2007. All patients in our study were males. No female patient was found. The youngest was a two weeks old male who was delivered by breech. No known etiological factor was found in 9 of the case. Four were diabetics; one patient was HIV sero positive while the other was the neonate who had a traumatic delivery.

Polymicrobial organism was isolated in all. No anaerobic culture was done. Four of the patients diedtwo diabetics, the HIV sero-positive patient and the neonate from severe sepsis. No skin grafting was performed. Key word: Fournier's gangrene
\end{abstract}

\section{Introduction.}

Fournier's gangrene (FG)is a rapidly progressive necrotizing fasciitis of the perineal and the genitourinary region(1), It is a rare but life threatening disease and a surgical emergency.2. It was first described by Alfred Jean Fournier in 1843/1844 in France.(3)) It was then referred to as idiopathic scrotal gangrene since he could not determine the etiology.

With advances in medicine, clinicians have vigorously searched for the aetio-pathologic causes of this lethal condition and associated it with a synergic polymicrobial infection of the perirectal, perianeal and genital region and occurring in both genders.(1).

Diagnosis is usually on clinical ground.(4) and investigations are essential to define the cause of an episode but not for the diagnosis of the disease(4). Prognosis depends on early diagnosis, the use of a broadspectrum antibiotics, and aggressive repetitive surgery. $(4,5)$

This retrospective study of fifteen cases of Fournier's gangrene and literature review is aimed at determining the features, and etiology, and the treatment regime offered in our environment.

\section{Patient and methods:}

Fifteen case notes of patient seen and treated with Fournier's gangrene in our facility(NDUTH) from 2002-2007 were reviewed. Patient age, sex, social class, occupation, educational status, were sought and documented from the case records. Also sought for were the site and extent of disease, associated illness, eg diabetis melitus, and and any other co-morbid condition.

The result of investigations were reviewed and the treatment given and the out- come of therapy were also noted. the study.

All patients who had severe local infection such as anal abscess, perirectal abscess, were excluded from

\section{Result:}

In our study, a total of 15 cases were seen. They were all males, no females was seen. Their ages ranges from 6 weeks to 75 years with mean age of 61 years. All the patients presented late to the hospital. They all had had one form of antibiotic or the other before presenting. There were no identifiable causes in $60 \%(9)$ cases. Three patients $(20 \%)$ were diabetic, Two $(13.33 \%)$ had local injury to the scrotum, while one $(6.67 \%)$ was sero-positive to Hiv and the other was the neonate who had a traumatic breech delivery. 10 of the cases were fishermen and farmers. This is the most common occupation of our people. Six of the patient had more than one wife and they were sexually active. 8 of them took alcohol and two admitted to smoking cigeratte. 


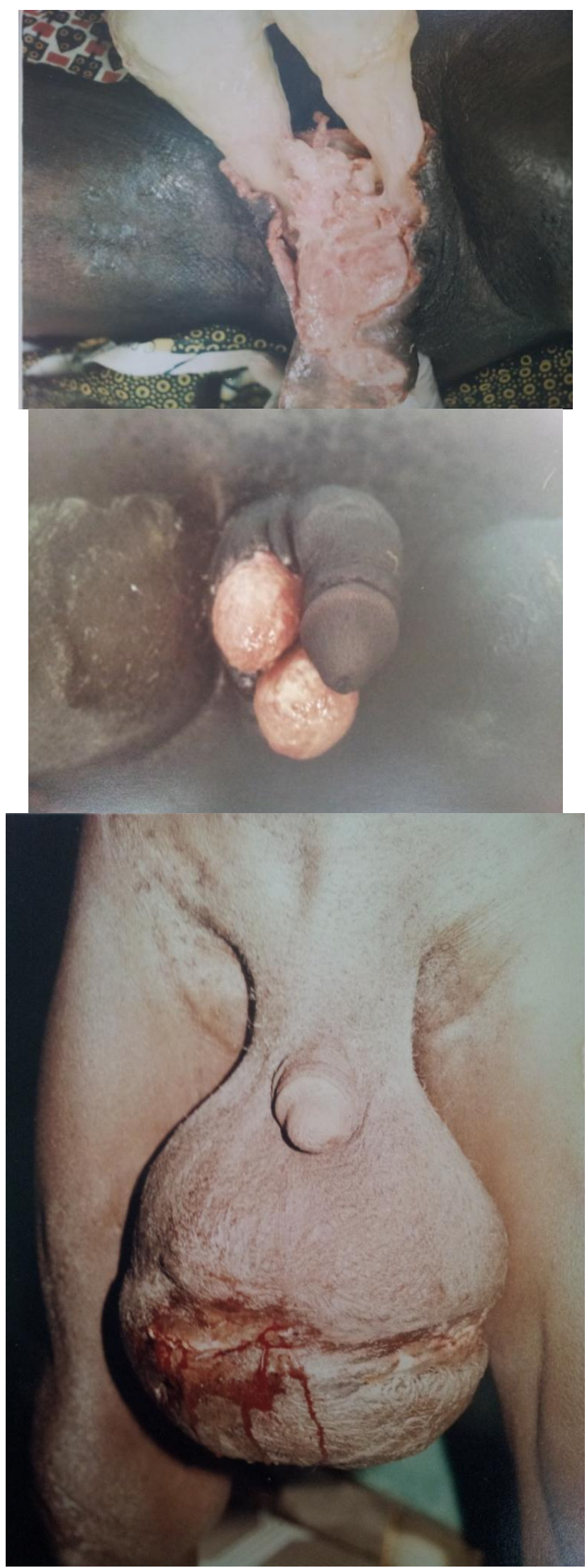



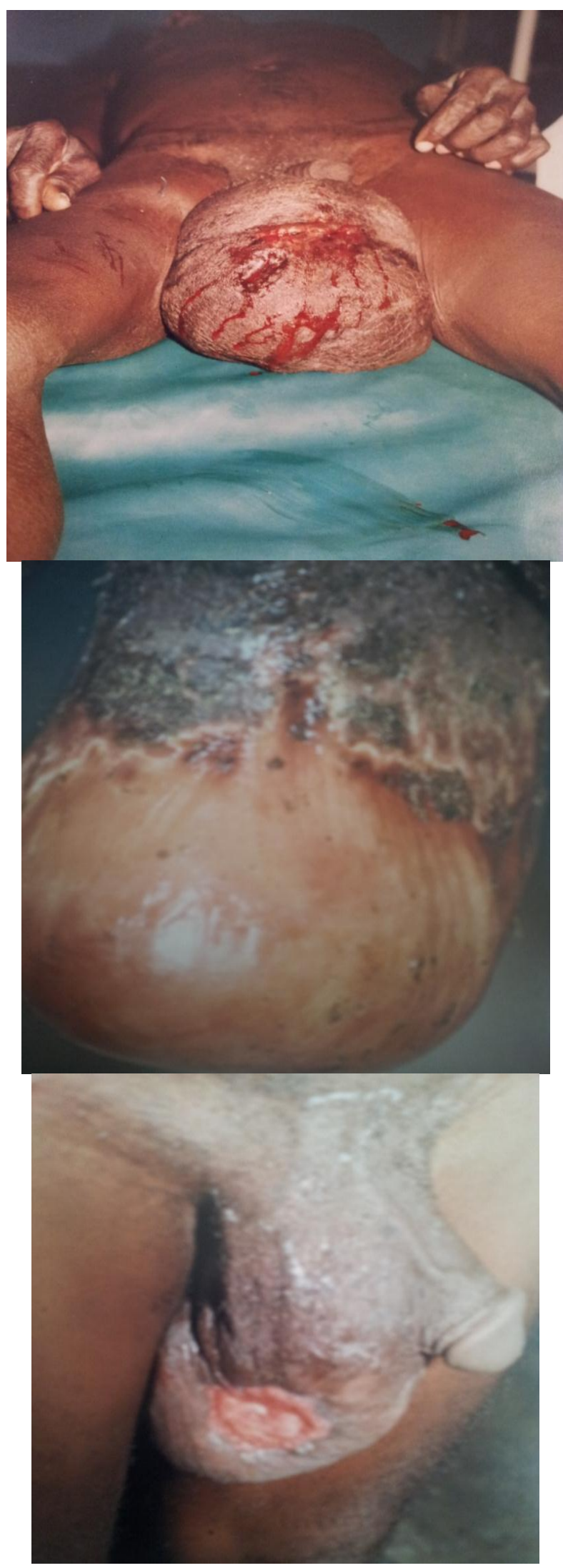


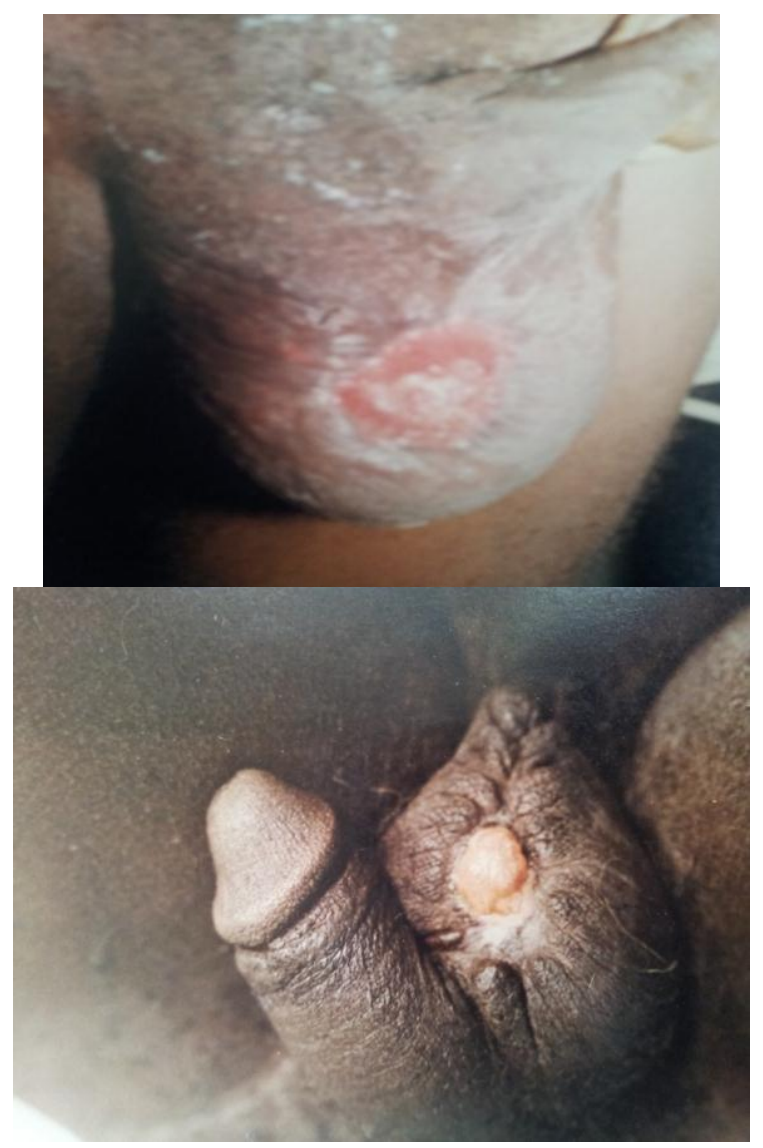

All patient presented late to the hospital. The earliest presented four days after commencement of symptom. They all had fever, pain and discharge of sero-purulent material from the site. They also had swelling, redness, tenderness, and black dermal necrosis of the skin.

Trauma to the groin was seen in only two cases including the neonate. Diagnosis was made on clinical ground. All had had one form of treatment including antibiotics before presenting. All also showed evidence of polymicrobial growth which was sensitive to gentamycine and ciproxine. All were resistance to ampicillin and Ampiclox. No anaerobic cultures were done in all.

The scrotum was involved in all the cases. Three patients had extension of disease to the perianal, penile and anterior abdominal wall. Two had exposed testes which were spared in all the cases.

They all had crystalloid infusion(resuscitation), aggressive repetitive wound debridement, analgesics, antibiotic and tetanus prophylaxis. Three of the patient had blood transfused. No skin grafting was done.

They all stayed more than three weeks in the hospital. The shortest stay was three weeks while the longest spent 12 weeks in the hospital.

Four (26.67\%) of the patients died, the rest were discharged home. Only seven out of the 15 cases came back for follow up.

\section{Discussion}

Fournier's gangrene is a life threatening disease and a medical emergency.(6) This lethal though rare condition was first described by Fournier in 1843/1844 as an idiopathic gangrene in relation to the scrotum. However, Meleney, in his article described it in relation to haemolytic streptococcus infection(7)), while Cullen associated it with bacteria acting in synergism.(8). In 1952, Walson et al, introduced the term necrotizing fasciitis.(9). Based on advancement in medicine, it is known that the disease is neither idiopathic nor spontaneous.(4).It is an infective gangrene which involves the subcutaneous fascia and part of deep fascia, and may involve any part of the body.(4).

It is a fatal disease that is world-wide in distribution. Though, disease is commoner in older age group, but no age is exempted(10,11), as was demonstrated in our series. In our study, the oldest patient was a 75 year old while the youngest was a neonate who had a traumatic breach delivery by a traditional birth attendant. The age distribution is in keeping with the report from other studies.

Most series of Fournier's gangrene have reported a 10: 1 male to female ratio.(12). In the female, the labia majoria and perianal regions are involved. It is said to take the same fulminating course as that of the male, but 
in our series, no woman was seen. Underreporting or the inability of physicians to recorgnize the occurrence of FG in the female may be responsible.

The majority of patient with Fournier's gangrene were thought to be immunosuppressed and associated with several co-morbid and etiological factors,(13) thus the primary wound might have been minor or might have arisen from an otherwise uneventful operation. Although this theory might be true, but our series does not support this assertion. The only common factor among the patients studied was that they were fishermen and farmers and they all consume alcohol. The consumption of alcohol is common in this part of the world and cannot be linked as a causative factor to FG. Only six patients(40\%) had an identifiable co-morbid conditions. (The neonate who had a traumatic breach delivery by a traditional mid-wife, the three elderly men who had diabetes and the patient sero-positive to Hiv.) In report of other studies, DM was found in as much as $46 \%$ of the cases(5). Our figure however, was lower.

They were all sexually active and $5(20 \%)$ of them had more than one wife. This might explain to a lesser extent that the route of entry of the organism may be trivial and may be unnoticed by the patient.

In all the patients, pain was the initial presenting symptom. This was sudden in onset and became rapidly excruciation. Swelling, redness, tenderness and black dermal necrosis are typical cutaneous manisfestations. These were present in all the cases in various degrees, and agree with the report of other studies. Fever, malaise, and discharge of sero-purulent substance was also present in all the patient. These symptoms are therefore universal for all patients with FG.

Based on the wrong cultural believe and awareness, all patients under the study presented late to the clinic. This is also indicative of the poor socio economic status of the patients resulting in poor attendance at medical facilities. Poverty, ignorance, and spiritual belief are all contributory factors. This trend is likely to continue, unless an intense health public awareness campaign is made by the authorities involved.

The penis and anterior abdominal walls were involved in three cases. Those who had this extensive gangrene died from severe sepsis and circulatory collapse.

As reported in other studies, diagnosis was made on clinical ground in all the cases. $(2,12)$ however, radiological evaluation, including CT and MRI, provides early detection in clinically indolent cases(14) .

Various organism both aerobes and anaerobes have been identified as agents that acts synergistically in causing the disease. In our series, poly microbial organisms were identified $(2,4,15$. The most common organism was Escherichia coli, Staphylococcus aureus, Klebseilla pneumonia and Streptococcus, and fungus. This is agreement with results of other reports $(16,17,18)$ ) Although we did not culture anaerobes, we believe the result would have been the same with the report of other studies. We also believe that synergism of these different micro-organism might be responsible for the fulminant infection.

Four of the patients were toxic and collapsed as at the time of presentation. They were resuscitated with fluid, analgesics and managed with aggressive antibiotic therapy. They all had debridement done and they received nutritional support.

In spite of advancement in management, mortality rates are still high. In some series, it ranges from $14-45 \%(2,15,19)$. However, in this study, our mortality was $26.67 \%$. it was higher in those patient with identifiable cause for their pathology-Trauma, Diabetics and HIV seropositive. This figure though high, is in agreement with other reported cases.

The long stay of the patients in hospital is attributed to their late presentation, and inability to provide the prescribed drugs. Also the poor follow up of patients is due to ignorance. Patients do not see the need to come back to the hospital once they are well.

\section{Conclusion.}

Fournier's gangrene is necrotizing fasciitis and is usually due to mixed infection.

Early presentation and diagnosis, supportive measures and the use of broad spectrum antibiotic and aggressive surgical debridement remain the standard of management of patient with Fournier's gangrene.

\section{References.}

[1]. Morpurgo E. Fournier's gangrene. Surg Clin N Am 2002;82: 1213-24

[2]. Shi-Guo L, Hong-Hwa C et al. Fournier's gangrene in female patients. J Soc Colon Rectal Surgeon(Taiwan) 2008; $19: 57-62$.

[3]. Fournier's J A. Gangrene foudroyant de loa verg. Med Pract Paris 1883; 4: 589-597.

[4]. Efem SE. The features and eatiology of Fournier's gangrene. Postgrad Med J 1994, 70,568-571.

[5]. Yanar H, Taviloalu K, et al. Fournier's gangrene: risk factors and strategies for management. World J Surg, 2006 ; $30(9)$ : $1750-4$.

[6]. Katusic J, Stimac G et al. management of Fournier's gangrene: case report and literature review. Acta Clin 2010; 49(4): 453-7.

[7]. Meleny F L. Haemolytic streptococcal gangrene. Arch Surg 1924, 9: 317-364.

[8]. Cullen T S .Progressive enlarging ulcers of abdominal wall involving the skin and fat, following drainage of abdominal abscess apparently of appendical origin. Sur Gynecol Obstet, 1924, 38: 579-582.

[9]. Wilson B. Necrotising fasciitis. Am J Surg 1953 18: 416-431.

[10]. Adams J R, Jr Mata JA ea al. Fournier's gangrene in children. Urology 1990, 35: 439-441.

[11]. Silva-Vilasenor J A, Velazquez M et al. Fournier's gangrene in a new born. Bol Med Hosp Infant Mex 1990, 47: 48- 50.

[12]. Eke N, Fournier's gangrene: a review of 1726 cases. Br J Surg, 2000, 87: 718-728. 
[13]. Gurdal M. Predisposing factors and treatment outcome in Fournier's gangrene. Urol Int 2003;70(4): 286-90.

[14]. Sherman J. Early CT findings of Fournier's gangrene in a healthy male. Clin Imaging 1998;22(6):425-7.

[15]. David P, Girvan MD et al. Fournier's gangrene. Canadian J of surg 1996; 39:448-449.

[16]. Fahal, AH, Hassan MA, Fournier's gangrene in Khartoum, Sudan. Br J Urol 1988, 61: 451-454.

[17]. Wolach MD, MacDermott JP, et al. treatment and complications of Fournier's gangrene. Br J Urol 1989, 64: 310-314.

[18]. Campos J A, Martos J A et al. Synchronous caverno-spongious thrombosis and Fournier's gangrene. Arch Esp Urol 1990, 43: 423426.

[19]. Vick R. Fournier's gangrene. Urol Clin North Am 1999; 26(4): 841-9. 